\title{
Classification of common household plastic wastes combining multiple methods based on near-infrared spectroscopy
}

\author{
Qinyuan Duan, Jia Li ${ }^{*}$
}

China-UK Low Carbon College, Shanghai Jiao Tong University, 3 Yinlian Road, Shanghai, 201306, People's Republic of China

*Corresponding author: Jia Li (weee@sjtu.edu.cn)

\author{
Supplementary Information (SI) Content \\ 9 pages (including the cover page) \\ Details about the classification methods applied (Page S5-S6) \\ 7 Figures 2 Tables References (Page S9)
}

Figure S1. Waste plastic samples of 7 types according to resin identification code developed by the American Society of Plastics Industry.

Figure S2. NIR spectrum acquisition platform.

Figure S3. Preprocessing methods in comparison on the spectra of virgin PP.

Figure S4. Preprocessing methods in comparison on the spectra of waste PP.

Figure S5. First-derivative spectra of 7 types (smoothed by S-G filter).

Figure S6. plastic wastes sorting device

Figure S7. The working process of air nozzles

Table S1. Accuracy in the validation of the classifiers for HDPE and LDPE.

Table S2. Accuracy in the validation of the classifiers for PET and PC. 


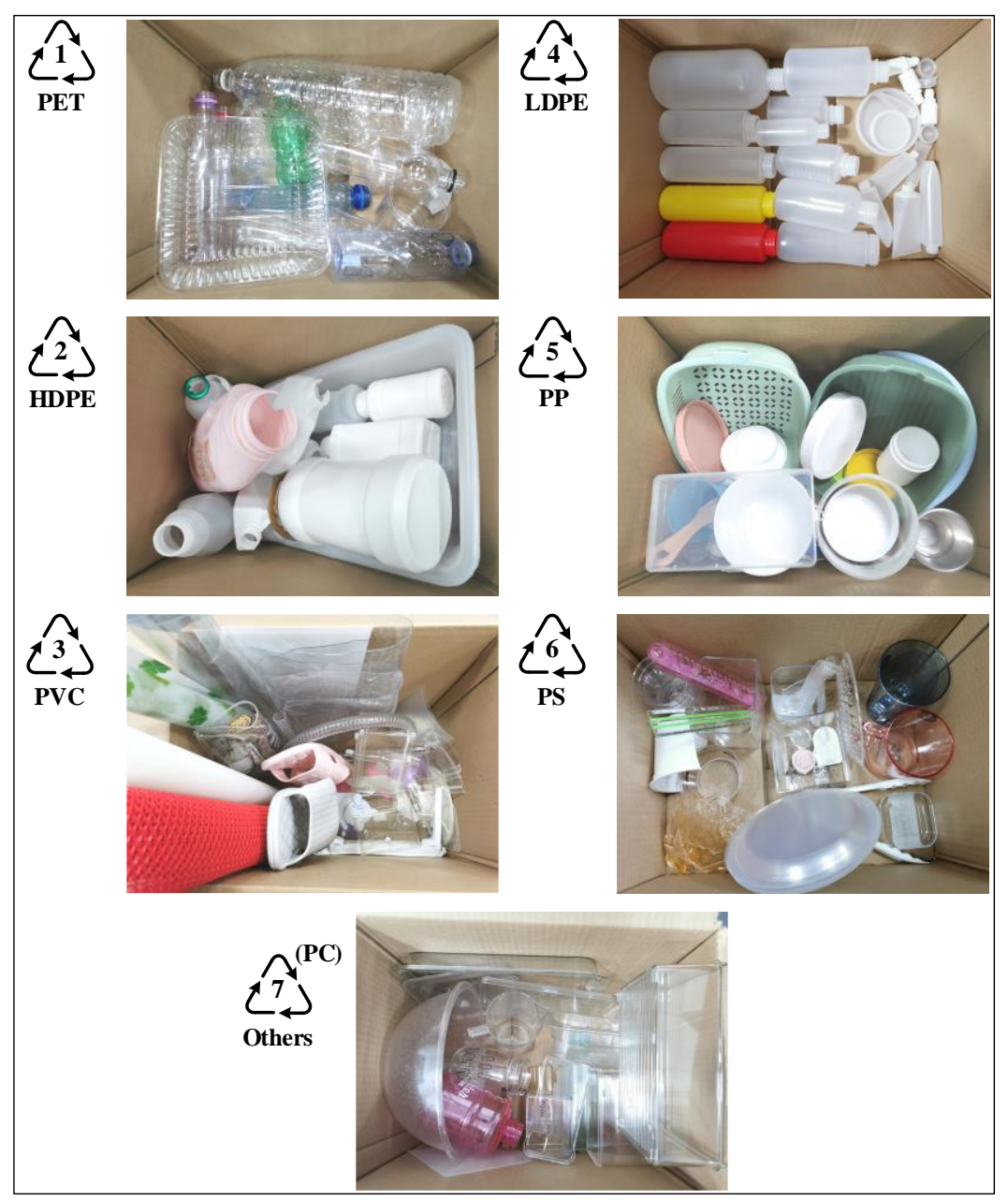

Figure S1. Waste plastic samples of 7 types according to resin identification code developed by the American Society of Plastics Industry

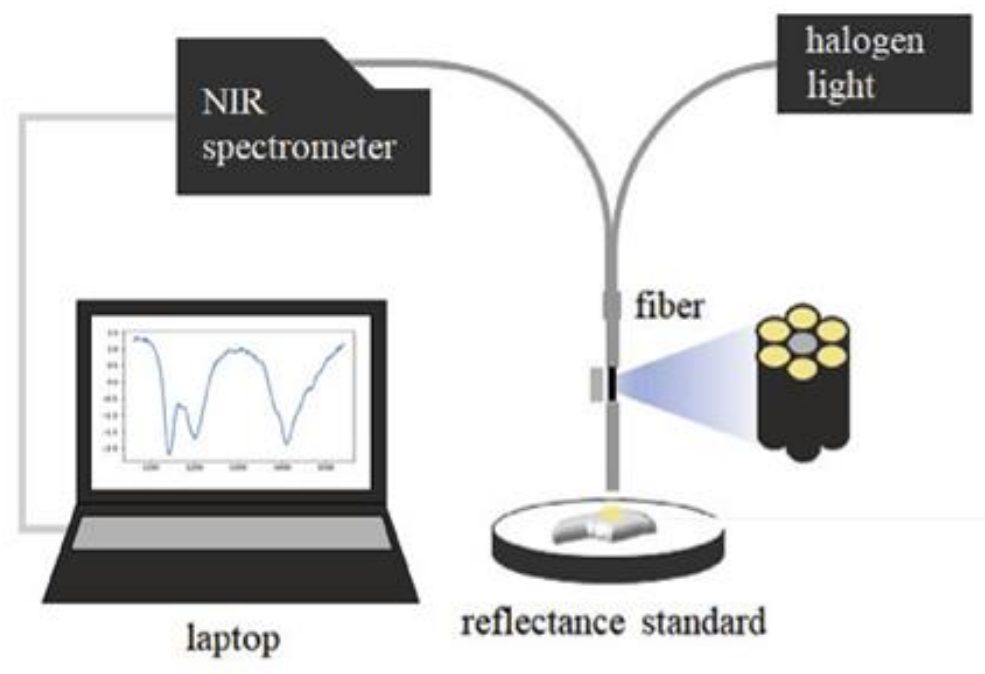

Figure S2. NIR spectrum acquisition platform ${ }^{1}$ 

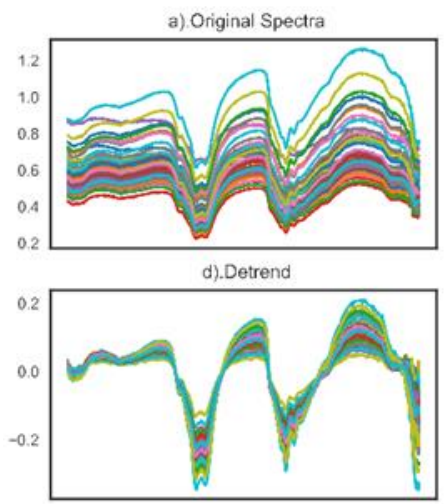

g). 1 st Derivative

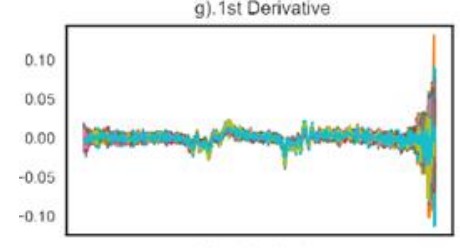

j).2nd Derivative

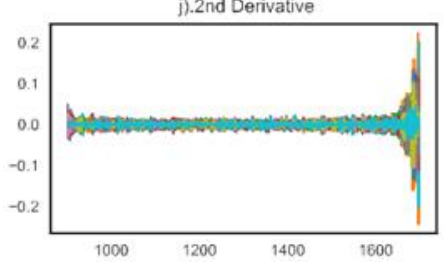

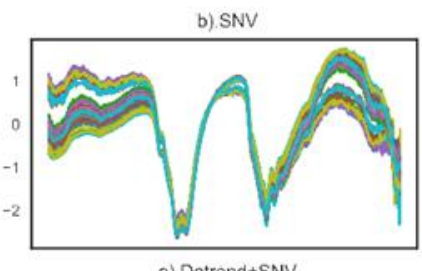

e). Detrend+SNV

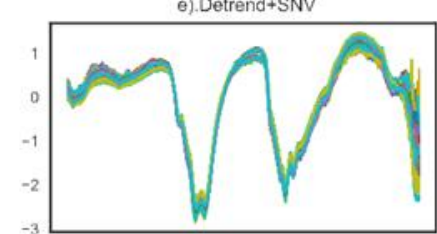

h). 1 st Derivative+SNV

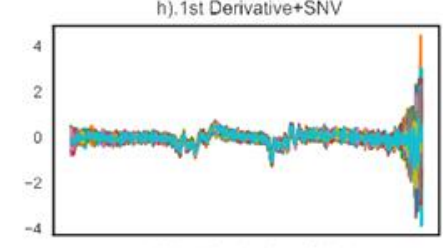

k).2nd Derivative+SNV

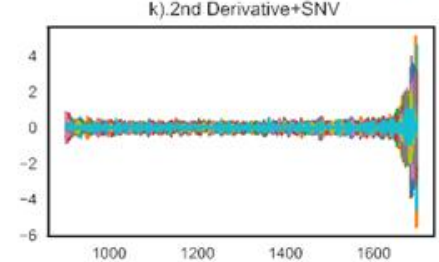

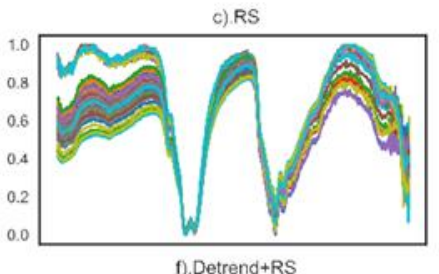

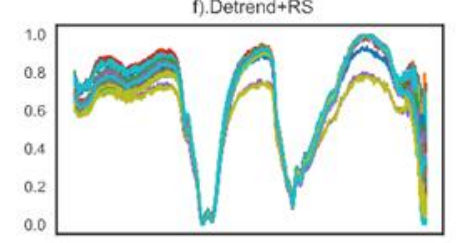

i).1st Derivative+RS

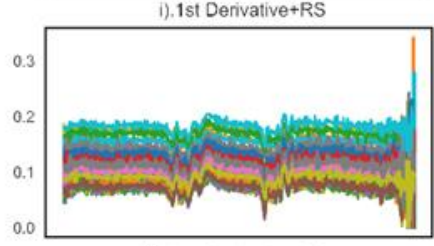

1).2nd Derivative+RS

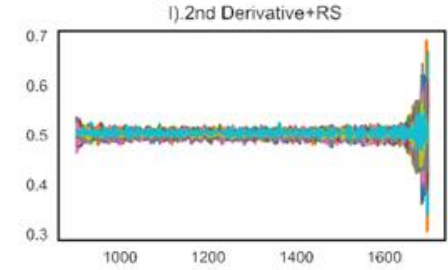

Figure S3. Preprocessing methods in comparison on the spectra of virgin PP. a)

original spectra; b) SNV; c) RS; d) detrend; e) detrend+SNV; f) detrend+RS; g) firstderivative; h) first-derivative+SNV; i) first-derivative+RS; j) second-derivative; k) second-derivative $+\mathrm{SNV}$; 1) second-derivative $+\mathrm{RS}$ 


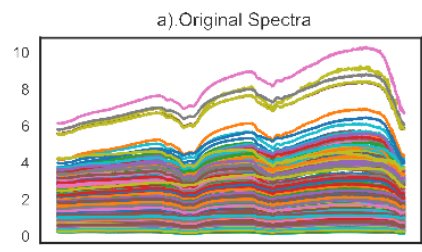

d). Detrend

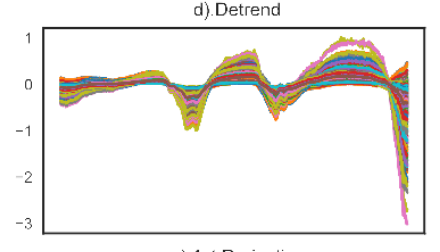

g).1st Derivative

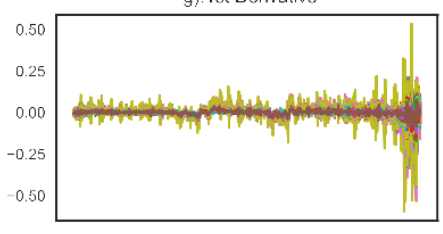

j).2nd Derivative

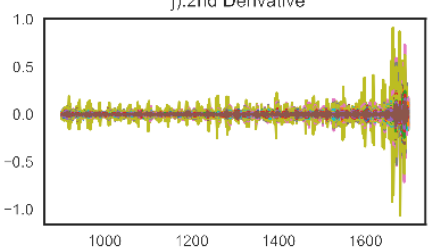

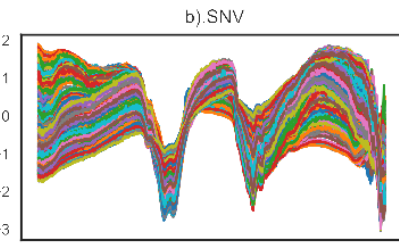

e). Detrend $+S N \backslash$

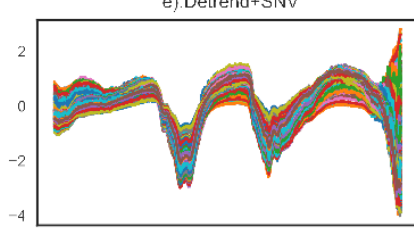

h). 1 st Derivative+SNV

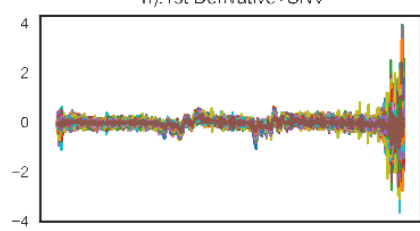

k).2nd Derivative+SN

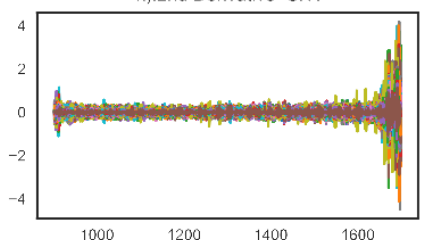

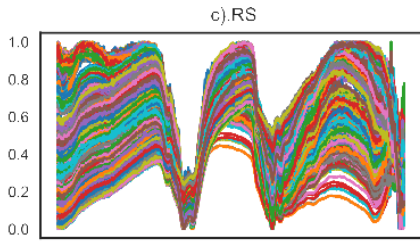

f). Detrend+RS

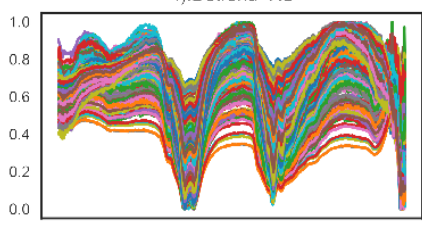

i).1st Derivative+RS

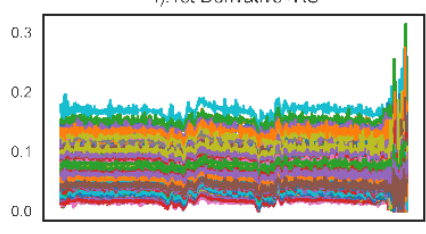

1).2nd Derivative+RS

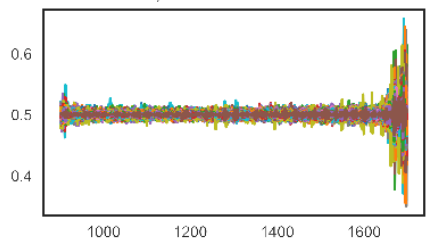

Figure S4. Preprocessing methods in comparison on the spectra of waste PP. a) original spectra; b) SNV; c) RS; d) detrend; e) detrend+SNV; f) detrend+RS; g) firstderivative; h) first-derivative $+\mathrm{SNV}$; i) first-derivative+RS; j) second-derivative; k) second-derivative+SNV; 1) second-derivative+RS; 


\section{Classification methods}

In the study, four classification methods were evaluated including LDA, SAM, SVM, and PLS-DA.

SAM is a classification method that comparing the cosine of the test spectrum with the reference spectrum directly. When the cosine value is close to 1 , it indicates that the test spectrum is highly similar to the reference spectrum. If the value is close to 0 , it means they are not similar contrarily.

PLS-DA is a linear classification method based on PLS regression. PLS regression can achieve regression and reduce the dimension of the data at the same time which differs from general linear regression. In order to determine the model parameter, 10fold cross-validation was applied and the root mean square error (RMSECV) of the cross-validation set of PLS regression was taken as the evaluation standard.

LDA is a classic algorithm of pattern recognition. The basic idea is to project the original data to a lower dimension in which the data points of each category are expected as close as possible and the distance between the category centers of different categories is as large as possible ${ }^{2}$.

SVM is a binary classification model working with supervised learning. It aims to find a linear segmentation defined in the feature space that maximizes the interval between different types of samples. In the study, Gaussian kernel was chosen and 10fold cross-validation was used, accuracy was the basis for evaluation to decide the parameters.

PCA is an unsupervised dimensionality reduction algorithm, it can find a new set of bases called the principal component (PC) that can best re-express the data set, and 
this set of bases is a linear combination of the original bases. Because of the high dimension of the original spectral data, it requires to be processed using PCA before classification applying LDA and SVM while it's unnecessary for the other two methods due to their properties.

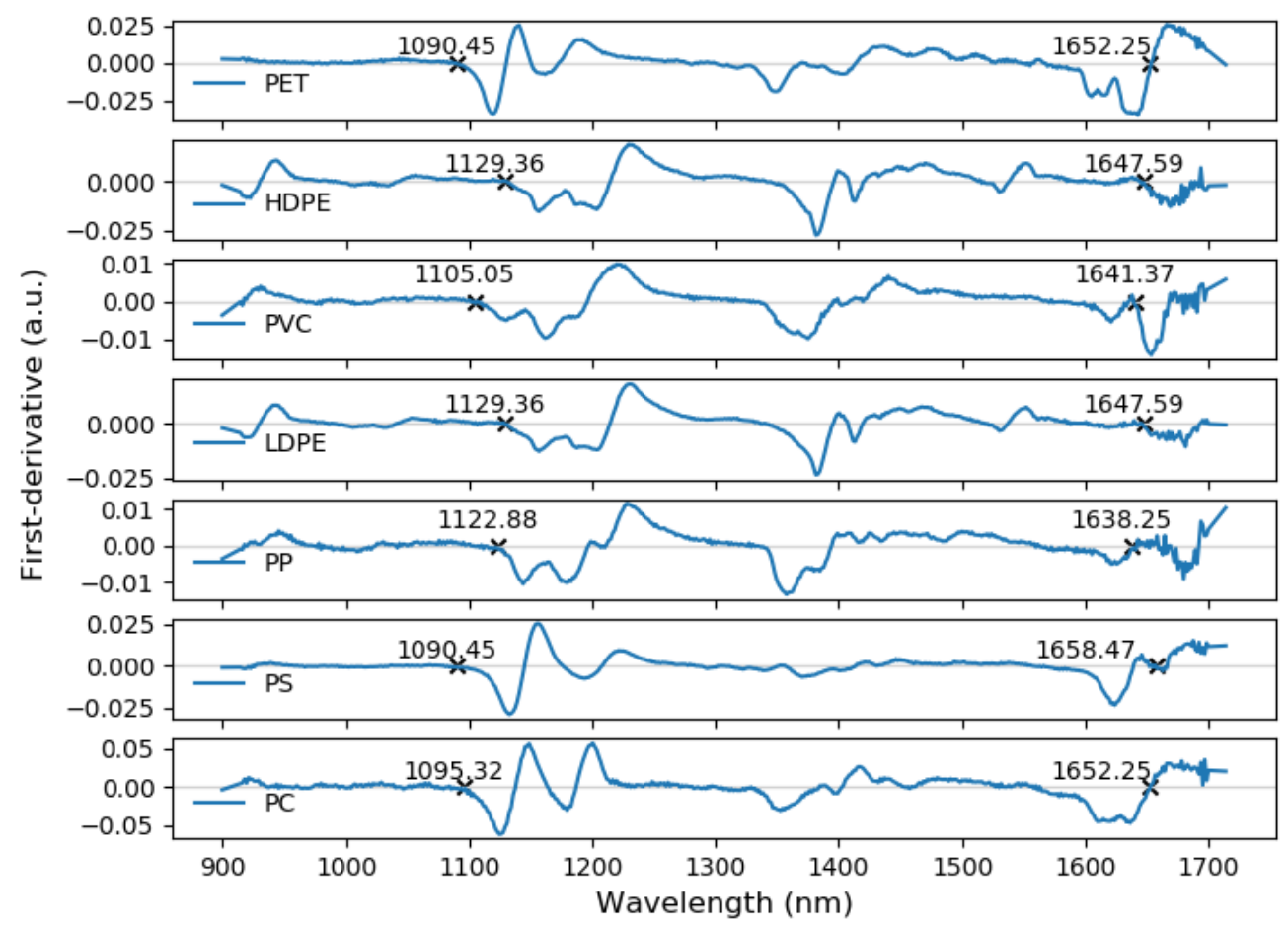

Figure S5. First-derivative spectra of 7 types (smoothed by S-G filter) 
Table S1. Accuracy in the validation of the classifiers for HDPE and LDPE (the virgin samples of each type were randomly divided into a training set and a test set at a ratio of $6: 4$, repeated 100 times, and the average values were taken as the final results)

\begin{tabular}{cccccc}
\hline & Band 1 & Band 2 & Band 3 & Band 4 & Band 5 \\
\hline PCA-LDA & 1.000 & 1.000 & 1.000 & 1.000 & 1.000 \\
PCA-SVM & 1.000 & 1.000 & 1.000 & 1.000 & 1.000 \\
SAM & 1.000 & 1.000 & 0.994 & 1.000 & 1.000 \\
PLS-DA & 1.000 & 1.000 & 1.000 & 1.000 & 1.000 \\
\hline
\end{tabular}

Table S2. Accuracy in the validation of the classifiers for PET and PC (the virgin samples of each type were randomly divided into a training set and a test set at a ratio of 6:4, repeated 100 times, and the average values were taken as the final results)

\begin{tabular}{cccccc}
\hline & Band 1 & Band 2 & Band 3 & Band 4 & Band 5 \\
\hline PCA-LDA & 1.000 & 1.000 & 1.000 & 1.000 & 1.000 \\
PCA-SVM & 1.000 & 1.000 & 1.000 & 1.000 & 1.000 \\
SAM & 1.000 & 1.000 & 1.000 & 1.000 & 1.000 \\
PLS-DA & 1.000 & 1.000 & 1.000 & 1.000 & 1.000 \\
\hline
\end{tabular}




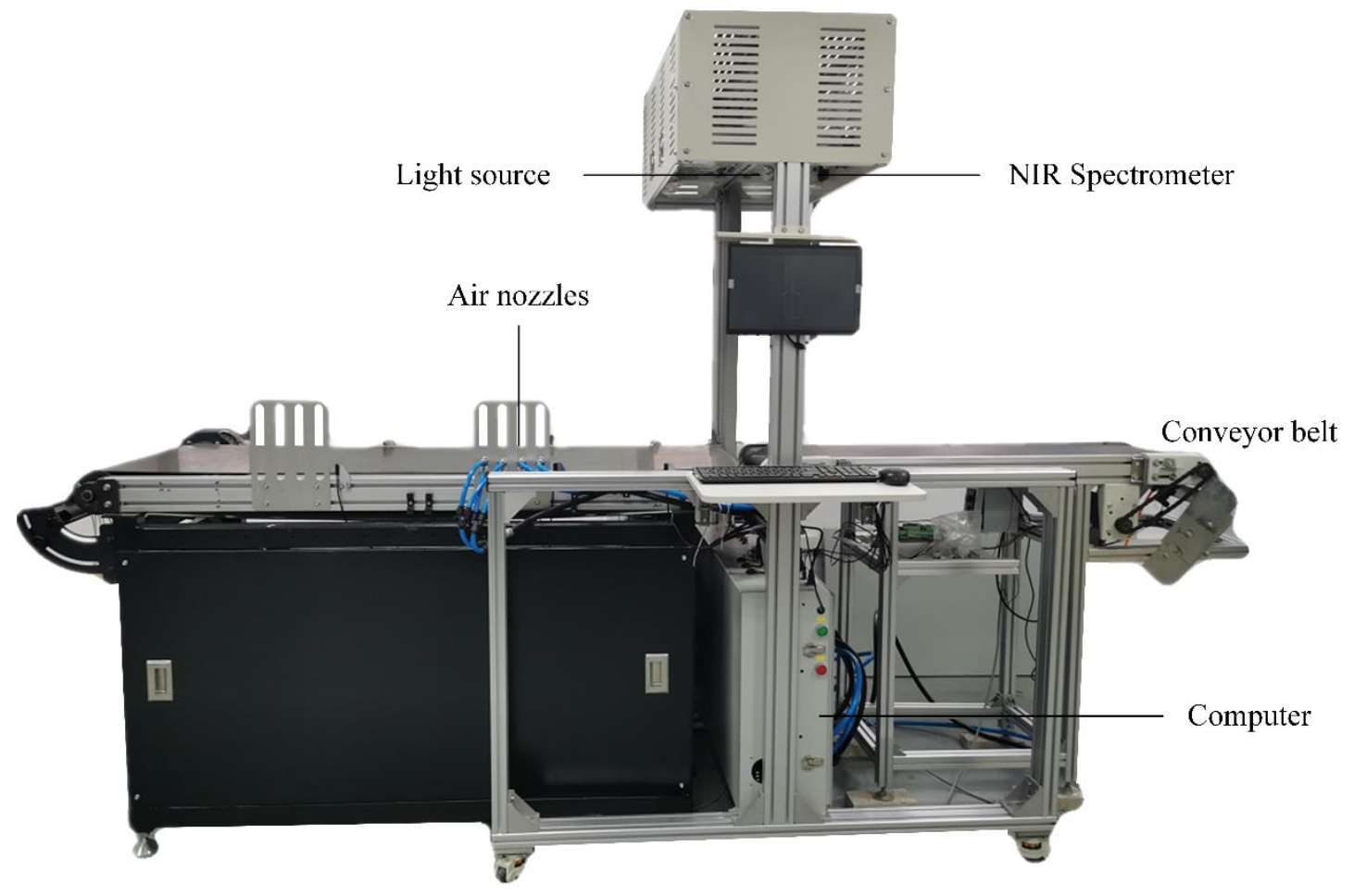

Figure S6. plastic wastes sorting device

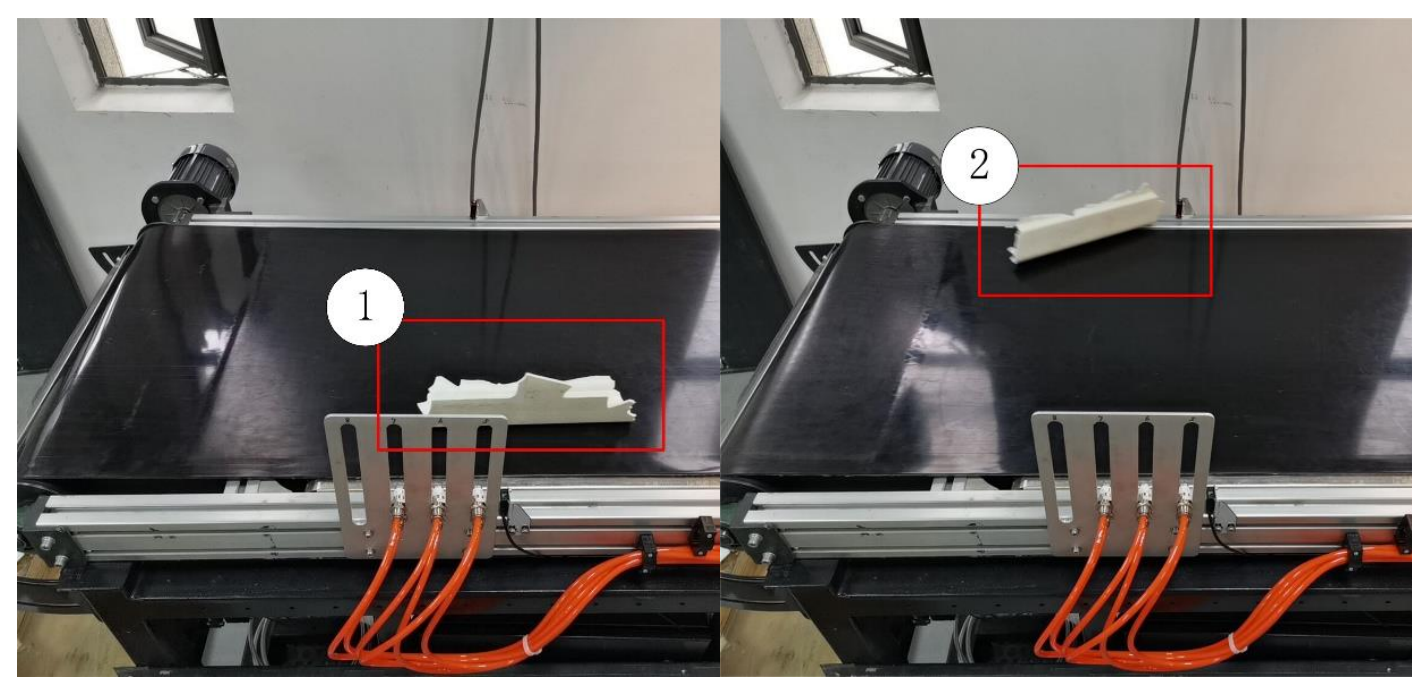

Figure S7. The working process of air nozzles (1) the identified material arrives at a specific location (2) the air nozzles eject compressed air 


\section{References}

1. Wu, X. Y.; Li, J.; Yao, L. P.; Xu, Z. M., Auto-sorting commonly recovered plastics from waste household appliances and electronics using near-infrared spectroscopy. Journal of Cleaner Production 2020, 246.

2. Tharwat, A.; Gaber, T.; Ibrahim, A.; Hassanien, A. E., Linear discriminant analysis: A detailed tutorial. A/ Commun. 2017, 30 (2), 169-190. 